\title{
Proceso de Bolonia (I): educación orientada a competencias
}

\author{
J. Palés-Argullós ${ }^{a, b}$, M. Nolla-Domenjó ${ }^{a, c}$, A. Oriol-Bosch ${ }^{a}, A$. Gual ${ }^{a, b}$
}

'The hidden curriculum is a set of influences that function at a level of organizational structure and culture, affecting the nature of learning, professional interactions and practice.'

The Association of Faculties of Medicine of Canada [1]

El proceso de Bolonia se inició en 1999 con un compromiso político de los ministros de educación de cuatro países en Paris y se concretó un año después en la 'Declaración de Bolonia. Este acuerdo puso en marcha un trascendente proceso de transformación educativa que impone a las universidades del Espacio Europeo de Educación Superior la obligación de orientar la educación de grado a la obtención de competencias [2]. Para algunos países, y en especial en el ámbito de la educación médica, no fue un proceso nuevo porque la educación orientada a la obtención de competencias es lo que los anglosajones denominaban outcome-based education, cambio educativo que en 1996 implementó por primera vez la Facultad de Medicina de la Brown University [3]. Con esta estructura se pretendía superar el antiguo sistema caracterizado por el mosaicismo de los currículos organizados en materias o asignaturas, y se centraban en la innovación de los procesos educativos. Los objetivos educativos, declaración de intenciones, eran sustituidos por resultados del aprendizaje de los dominios competenciales ( $\mathrm{Ta}$ bla I) que debían ser constatados (evaluados). La capacidad 'de hacer' alcanzada por los graduados devenía el objetivo esencial a conseguir, siendo el cómo se alcanzarán función de su eficacia y eficiencia en lograrlo. Dejaba de importar cómo se criaba el gato, y sí, que cazara ratones.

\section{Competencia/s}

El concepto de competencia requiere, cuando se utiliza normativamente como en el caso del proceso de Bolonia y de la legislación que ha generado, una definición precisa que no presente ambigüedades ni genere confusiones. En su acepción cuarta, la Real Academia Española define competencia como 'aptitud,' 'idoneidad', y en su tercera acepción, como 'incumbencia' [4]. Las competencias tal como las entiende la educación orientada a competencias no se referencia tanto al ámbito como a la aptitud. Es obvio que este concepto, para poder ser de utilidad operativa, requiere ser definido con mayor precisión.

Las competencias, según las definió en 2005 la Comisión Europea, representan una combinación dinámica de atributos, habilidades y actitudes. Los resultados de aprendizaje finales de un período formativo se expresan en forma de competencias. El grupo de Roma, en su glosario terminológico (2008), define competencia como 'conjunto de conocimientos, habilidades, actitudes, comportamientos y actuaciones -performance- de un profesional'. La Ley de Cohesión y Calidad del Sistema Nacional de Salud (Ley 16/2003), en su capítulo III, define competencia profesional como 'la aptitud del profesional sanitario para integrar y aplicar los conocimientos, habilidades y actitudes asociadas a las buenas prácticas de su profesión para resolver los problemas que se le plantean' [5] y posteriormente, la Ley de Ordenación de las Profesiones Sanitarias (Ley 44/2003) determina la constitución de un sistema de reconocimiento del desarrollo de dichas competencias. Existe pues un marco normativo para las competencias profesio-
Fundación Educación Médica (FEM).

b Facultad de Medicina. Universitat de Barcelona. Fundació Doctor Robert. Universitat Autònoma de Barcelona.

Correspondencia Dr. Jordi Palés Argullós. Departamento de Ciencias Fisiológicas I. Facultad de Medicina. Universitat de Barcelona. Casanova, 143. E-08036 Barcelona.

E-mail jpales@ub.edu 


\section{Tabla I. Habilidades (competencias) evaluables según la Brown University Medical School [3].}

Habilidades clínicas básicas

Aplicación de las ciencias básicas en la práctica médica

Diagnóstico, manejo y prevención

Aprendizaje a lo largo de la vida

Autoconciencia, autocuidado y crecimiento personal

Contacto social y comunitario de la asistencia

Razonamiento moral y ética clínica

Solución de problemas

nales y su desarrollo y, además, un imperativo de orientar la educación hacia ellas [6].

Sin embargo, la tarea no es sencilla por cuanto las competencias profesionales son capacidades construidas con componentes cognitivos que, combinados con habilidades psicomotoras y actitudes basadas en valores y creencias, se expresan en conductas coherentes y previsibles. Las competencias son las que posibilitan los desempeños adecuados y se expresan a través de ellos, pero sólo son los atributos necesarios que los hacen posibles, existiendo elementos en el entorno que pueden afectar sensiblemente los desempeños. Por otra parte, no deben confundirse los comportamientos observables con las competencias que los posibilitan.

En las últimas décadas, el ámbito anglosajón ha llevado a cabo distintas aproximaciones a la taxonomía de las competencias de las profesiones sanitarias. En nuestro círculo más inmediato encontramos, en la segunda mitad de la década de los noventa, que el Consell Català d'Especialitats en Ciències de la Salut (CCECS) [7] hizo una aproximación de los atributos necesarios para la realización de las grandes tareas que las diferentes profesiones sanitarias llevaban a cabo. El CCECS aprobó, con el consenso de los respectivos colegios profesionales, las competencias de la profesión de enfermería (1998), las de los médi-
Tabla II. Taxonomía de las competencias médicas, según el CCECS [7].

Competencias relacionadas con el encuentro médico-paciente: anamnesis y exploración

Competencias relacionadas con la definición de problemas y la formulación de hipótesis

Competencias relacionadas con el 'triaje' de pacientes

Competencias relacionadas con los procedimientos y con situaciones clínicas específicas

Competencias relacionadas con la identificación y valoración de los factores psicológicos

Comunicación interpersonal

con el paciente y sus familiares

Evaluación de los factores sociales

Comunicación y colaboración entre colegas

Competencias relacionadas con la evaluación de riesgos y la promoción de la salud

Dominio de la ética, los valores y la legalidad

Competencias relacionadas con el mantenimiento y la mejora de las capacidades profesionales

Competencias relacionadas con la gestión clínica

cos (2000), las de los odontólogos y farmacéuticos (2001) y las de los psicólogos clínicos y salubristas (2002). La taxonomía de las competencias médicas, ordenadas según funciones o tareas esenciales, se expresó en términos de capacidades de desempeño (Tabla II). En estos dominios se identificaban 93 capacidades, sin incluir los procedimientos. Las competencias identificadas podían, por la naturaleza de su definición, ser observadas directa o indirectamente y, en consecuencia, todas las competencias definidas eran evaluables con algún instrumento de medida.

En Gran Bretaña, en 1993, el General Medical Council elaboró un documento con recomendaciones para las facultades de medicina cuya versión más reciente es del año 2009 [8,9]. En él se explicitan las capacidades del graduado que las facultades de medicina deben formar, ordenadas en tres ámbitos -como científico, como clínico y como profesional-, para poder actuar dentro de los estándares 


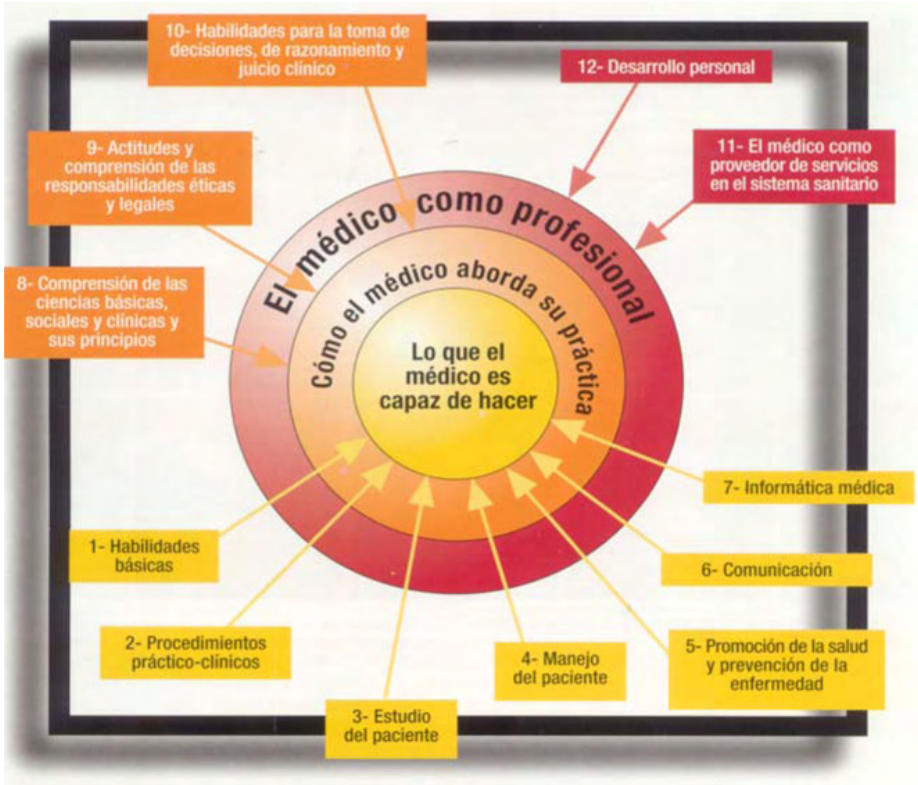

Figura 1. Competencias definidas por las facultades de medicina escocesas (adaptado de [10,11]). Portada de Educ Med 2001, vol. 4, n.0 1 .

establecidos en la guía de la buena práctica médica. El documento describe 105 capacidades de actuación sin enumerar los procedimientos.

En el año 2000, las cinco facultades de medicina de Escocia (Aberdeen, Dundee, Edimburgo, Glasgow y Saint Andrews) constituyeron un grupo de trabajo formado por sus respectivos decanos con el fin de elaborar un catálogo común de 'resultados de aprendizaje' (learning outcomes), basándose en el documento Tomorrow's Doctor de 1993 y 2009 del General Medical Council. El documento, denominado Learning Outcomes for the Medical Undergraduate in Scotland [10] y conocido como Scottish Doctor, se ha publicado actualmente en el 2009 en su tercera edición (Fig. 1); en él se distinguen tres niveles de competencias y, para cada nivel, diferente número de campos o dominios (Tabla III).

En el año 2003, el mismo grupo elaboró un segundo documento en el que trataba los diferentes métodos para evaluar las competencias (Learning Outcomes for the Medical Undergraduate in Scotland Phase II Project: Assessment) [11].

En el 2001, con el fin de promover una mejora de su currículo y de responder al reto del proceso de Bolonia, la Facultad de Medicina de la Universitat de Barcelona, a través de un grupo de trabajo, inició el proceso de definición de sus propios re- sultados de aprendizaje/competencias de acuerdo con el modelo del Scottish Doctor y teniendo en cuenta la opinión de diversos agentes (académicos, estudiantes, tutores de posgrado, colegios profesionales, sistema de salud, etc.) $[12,13]$, siendo este catálogo de competencias finales el primero desarrollado por una facultad de medicina española. Posteriormente, el mismo grupo procedió a realizar una valoración del grado de adquisición de dichas competencias mediante un cuestionario de autoevaluación a los estudiantes de último año de la licenciatura, poniéndose de manifiesto fortalezas y debilidades del plan de estudios [14].

También en 2003, la Facultad de Medicina de la Universitat de Lleida elaboró su propio catálogo de competencias, tomando como modelo el documento del Scottish Doctor [15].

Posteriormente, otras facultades de medicina definieron sus propias competencias basándose en distintos modelos, como la Universidad Complutense de Madrid [16].

La Agencia de Calidad para el Sistema Universitario en Cataluña (AQU) puso en marcha en el 2003 un grupo de trabajo para definir las competencias básicas comunes exigibles a los graduados en Medicina de las cuatro universidades catalanas [17]. Tomaron como modelo los dominios competenciales establecidos por el Institute for Inter- 
Tabla III. Niveles de competencias y sus respectivos campos o dominios, según las facultades de medicina escocesas [10].

Lo que el médico ha de ser capaz de hacer (hacer lo que es correcto, que equivaldría a las competencias técnicas):

- Habilidades clínicas

- Procedimientos prácticos

- Estudio del paciente

- Cómo tratar el enfermo

- Promoción de la salud y prevención de la enfermedad

- Habilidades de comunicación

- Habilidades para obtener información (informática, base de datos, etc.)

De qué manera aborda el médico su práctica (hacer lo que es correcto de manera correcta, que equivaldría a las competencias académicas, nivel conceptual o de pensamiento crítico):

- Comprensión de las ciencias básicas, clínicas y sociales y los principios en que se fundamentan

- Actitudes adecuadas, comprensión de las responsabilidades éticas y legales

- Habilidades para la toma de decisiones, análisis, razonamiento y juicio clínico

El médico como profesional (lo que es correcto, hecho de manera correcta por la persona correcta, que equivaldría a las competencias de desarrollo personal)

- El médico como proveedor de servicios en el sistema sanitario

- Competencias de desarrollo personal

national Medical Education (IIME) [18] (Fig. 2) y definieron 67 competencias correspondientes a siete dominios (Tabla IV).

Por su parte, la red temática de Educación Médica en Europa (MEDINE), y en concreto su grupo de trabajo I sobre el proyecto Tuning de medicina, definió los resultados de aprendizaje para el graduado europeo [19]. El grupo de trabajo en QA (Quality Assurance) de MEDINE, conjuntamente con la Federación Mundial de Educación Médica (WFME), adaptó los estándares de mejora de calidad a las condiciones europeas [20]. En el apartado 1.4 de los estándares de la formación básica del documento se establece que la facultad debe definir tanto 'las competencias que los estudiantes deben demostrar poseer para su graduación' como 'las conexiones de dichas competencias con las que se deberán adquirir en la formación posgraduada.' Además, el documento puntualiza que 'competencias en medicina ... incluiría el conocimiento y la comprensión de las ciencias básicas, clínicas, de la conducta y sociales, incluyendo salud pública y medicina de poblaciones y la ética médica relevante para el ejercicio de la medicina, actitudes y habilidades clínicas ... y la capacidad para el aprendizaje a lo largo de la vida y el desarrollo profesional'.

La Conferencia Nacional de Decanos de Facultades de Medicina españolas (CNDE) publicó en el 2005 el Libro Blanco de la Titulación de Medicina, como resultado de un proyecto financiado por la II Convocatoria de la Agencia Nacional de Evaluación y Acreditación de la Calidad (ANECA) para el diseño de planes de estudio y títulos de grado [21]. En el libro, la CNDE, además de analizar la situación de la enseñanza de la medicina en España y Europa, establecía un conjunto de competencias generales y especificas para el graduado en Medicina, inspirándose básicamente en los requisitos globales mínimos esenciales en educación médica del IIEM, y una vez realizadas consultas a organizaciones profesionales y científicas como la Organización Médica Colegial (OMC), la Federación de Asociaciones Científicas de Medicina (FACME), la Sociedad Española de Educación Médica (SEDEM), instituciones como el Ministerio de Sanidad y Consumo, el Consejo Nacio- 
Tabla IV. Dominios competenciales establecidos por el Institute for International Medical Education [18].

Valores profesionales, actitudes, comportamiento y ética

Fundamentos científicos de la medicina

Habilidades de comunicación

Habilidades clínicas

Salud de la población y los sistemas de salud

Utilización de la información

Pensamiento crítico e investigación

nal de Especialidades en Ciencias de la Salud, el Ministerio de Educación, Cultura y Deportes, el Consejo Estatal de Estudiantes de Medicina, y a expertos en educación médica. Los contenidos del Libro Blanco, especialmente las competencias definidas, fueron asumidas por la correspondiente orden ministerial (Orden ECI/332/2008, de 13 de febrero) [22] por la que se establecían los requisitos para la verificación de los estudios universitarios oficiales de Medicina como las competencias a adquirir por los graduados de Medicina en España. Sin embargo la confusión reinante entre conceptos diferentes, como 'conocimientos', 'habilidades', 'competencias', 'resultados de aprendizaje' y 'objetivos de aprendizaje', adquiere su máximo exponente en la referida orden al incluir indiscriminadamente, bajo el epígrafe de 'competencias', conceptos que corresponden unívocamente a 'conocimientos' o 'habilidades', o a verdaderas 'competencias'. Estos cinco términos, entre otros, se encuentran bien definidos en la bibliografía especializada y no se requiere una nueva definición. Sin embargo, al igual que hacemos en el mundo biomédico, en el ámbito de las ciencias de la educación debemos utilizar una terminología rigurosa, con las palabras apropiadas y con los mismos patrones científicos que en otras áreas de la ciencia.

La situación actual puede resumirse afirmando que el movimiento de la educación orientada a las

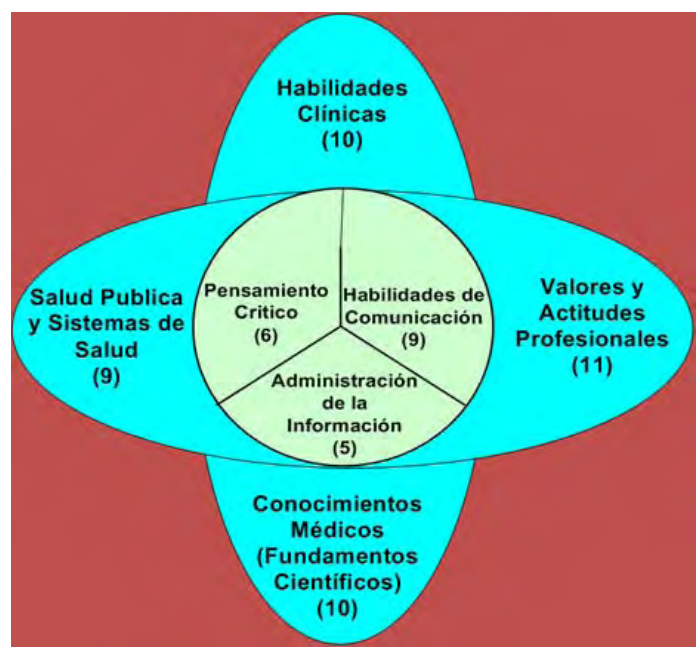

Figura 2. Requerimientos mínimos esenciales definidos por el Institute for International Medical Education (adaptado de [18]).

competencias, iniciado en Norteamérica a finales del siglo pasado (Project ACGME y CanMeds $[23,24]$, se encuentra actualmente en Europa en pleno apogeo evolutivo, enfrentándose a dos retos: las resistencias estructurales de las facultades y la necesidad de desarrollo del profesorado. Desde el punto de vista técnico sería necesario, por un lado, avanzar en la definición de los mínimos estándares exigibles en cada nivel en un contexto dado, y por otro, es urgente perfeccionar los instrumentos que permitan evaluar competencias de manera fiable y confiable. En cualquier caso, como se describe en el siguiente apartado, disponemos de instrumentos que permiten trabajar la evaluación de competencias y es necesario utilizarlos con rigurosidad y extender su uso entre nosotros.

\section{Evaluación de las competencias}

La educación orientada a las competencias que postula el proceso de Bolonia requiere una adaptación de los sistemas evaluativos en uso. Los llamados 'resultados de aprendizaje' no son meras declaraciones de intenciones de los responsables de los currículos, sino que se trata de competencias cuya adquisición debe comprobarse, es decir, evaluarse durante el proceso formativo. La eva- 
luación de las competencias exige disponer de instrumentos de cuantificación estandarizados, por un lado, y de un referente claro de lo que es un profesional competente, por otro.

A pesar de la aparición constante de nuevos instrumentos de medida hay que tener presente que ninguno por sí solo puede dar 'toda' la información sobre todos los componentes de la competencia profesional en general y ni siquiera de una competencia en particular. La evaluación de la competencia global de un profesional siempre debe combinar distintos instrumentos [25].

Recientemente, Ginsburg et al [26] han estudiado los criterios utilizados por los profesionales que evalúan la competencia clínica de los médicos en formación para detectar diferencias entre profesionales problemáticos y profesionales excelentes. Los ocho dominios utilizados por estos evaluadores clínicos se agrupan en la tabla $\mathrm{V}$.

Las características de la excelencia o insuficiencia de estos dominios se definieron en los términos siguientes:

- Conocimiento científico. La competencia en el domino del conocimiento científico viene determinada por la amplitud y la profundidad de la base de los conocimientos disponibles y por lo que se conoce como 'capacidad translacional', es decir, la habilidad para su utilización, basándose en evidencias, el juicio clínico o la capacidad para priorizar. Se ejemplifica positivamente porque la base de conocimiento es enciclopédica y la comprensión fisiopatológica es sorprendente, en tanto que su déficit se detecta por la superficialidad de las respuestas a las preguntas formuladas, la existencia de vacíos sorprendentes y la incapacidad para establecer diagnósticos diferenciales o planes de gestión clínica y priorización de las actividades.

- Profesionalismo. Se identifican cuatro áreas: ética de trabajo, habilidades laborales, respuesta al feedback y comportamiento. La confiabilidad sería una de las características de la ética de trabajo, que se expresaría en forma de puntualidad, conducta responsable, o su déficit con actitudes características del proletariado (ocupación de horario predeterminado). La capacidad organizativa sería la característica del área de las habilidades, expresadas en la buena gestión del tiempo y el control de la carga de trabajo, y su déficit, en señales como ilegibilidad de la escritura. En relación al feed-
Tabla V. Dominios utilizados para detectar diferencias entre profesionales problemáticos y profesionales excelentes [26].

Conocimiento científico

Profesionalismo

Interacción con los pacientes

Interacciones con el equipo de trabajo

Conocimiento del sistema de salud

Disposición (actitudes)

Confianza

Impacto causado (impresión que dan)

back, la información recibida es no tan sólo bien recibida, sino incluso buscada, generando respuesta de cambio adecuadas. Ignorar la información recibida, actuar defensivamente y no dar respuesta a las indicaciones son señales evidentes de déficit en esta área.

- Interacción con los pacientes. La apariencia y las formas de expresión (cortés, amistosa, profesional) son signos externos de comportamiento cuyos déficits visibles son las indumentarias inadecuadas o los comentarios derogatorios. La empatía es un atributo básico de la comunicación y la condición para establecer una buena relación con los pacientes, que se expresa mostrando interés por ellos como personas. Su déficit se muestra dando explicaciones poco claras, de forma confusa o de manera abrupta.

- Interacción con el equipo de trabajo. Se diferencia el comportamiento como miembro del equipo, del liderazgo y la relación con miembros de otras profesiones sanitarias. Las características del jugador de equipo son las buenas relaciones dentro de éste y la disposición a apoyar y ayudar a los compañeros, lo que se expresa asumiendo una proporción adecuada de la carga de trabajo y ofreciéndose a ayudar a los otros. Sus déficits se expresan con actitudes poco amigables, disruptivas de la dinámica del grupo, y maledicencias y actitudes conspirativas. 
Para el liderazgo se requiere capacidad de supervisión, educativa y de gestión del equipo, lo que representa tener la capacidad de ser un rol-model para los compañeros del equipo de trabajo, aprovechar las oportunidades para actuar como un educador, inspirar confianza y adaptarse a las posibilidades de los demás. Representa evitar actitudes autoritarias, no establecer planes sin consultar a los miembros del equipo, ni expresar actitudes dominantes en los detalles irrelevantes. En el equipo de trabajo no caben actitudes de falta de respeto por las opiniones de quienes no son médicos.

- Conocimiento del sistema de salud. El conocimiento del sistema sanitario y la capacidad de trabajar dentro de él es una competencia que exige saber siempre a quién se puede acudir, cómo conseguir que se hagan las cosas y cómo utilizar los recursos óptimamente.

- Disposición. La actitud percibida puede ser de carácter positivo (curiosidad, entusiasmo, energía puesta en lo que se hace...) o negativo (desconexión, pasividad, quejas acerca de las tareas encomendadas...) y basarse en características de la personalidad que también pueden ser de orden positivo (inteligente, brillante, listo) o negativo (desagradable, indolente, arrogante).

- Confianza. Se asienta en dos atributos: la credibilidad y el discernimiento. La credibilidad presupone tanto honestidad como lo que en inglés se conoce como reliability, es decir, que aquello esperado acaecerá (confiabilidad). Por lo tanto, puede creerse en lo que se dice, que no hay ocultación de errores ni es necesario hacer comprobaciones dobles con quienes se deposita confianza. El discernimiento se refiere al conocimiento de los propios límites sobre lo que se es capaz de hacer. Por tanto, se sabe cuándo hay que preguntar y su déficit se expresa por consultas demasiado frecuentes o muy tardías, así como por una falta de conciencia sobre las propias limitaciones.

- Impacto causado. La impresión dada a los supervisores puede ser de tipo positivo (fue un placer observar cómo trabajaba, lo hacía como lo habría hecho yo) o negativo (causó más trabajo que cualquier otro, fue una alegría perderlo de vista).

Lo curioso del caso es que tres de los dominios utilizados para evaluar la competencia clínica ni tan siquiera pueden considerarse competencias tal como las vienen definiendo los organismos responsables, y en realidad son sólo actitudes que expresan el carácter individual. Ello implica que existe la necesidad de avanzar en el diseño de un modelo evaluativo que permita establecer lo que sea relevante de medir y cómo integrarlo para evitar caer en una fragmentación que conduzca a la irrelevancia [27].

No se debe perder de vista que han aparecido nuevos instrumentos que han ampliado los aspectos de la competencia profesional susceptibles de evaluarse. Así, cuando se está en proceso de desarrollar nuevos instrumentos, su aplicación no sería legítima con un enfoque de evaluación de tipo sumativo, ya que para el estudiante esto puede tener consecuencias relevantes. Una evaluación tiene finalidades sumativas cuando los resultados son la base para la toma de decisiones sobre el nivel de competencia profesional (aprobado/suspenso, obtención de un título, obtención de una acreditación, etc.). En cambio, sí es recomendable usar los instrumentos en desarrollo con un enfoque formativo de la evaluación. Se dice que una evaluación tiene finalidades formativas cuando su objetivo fundamental consiste en proporcionar a los estudiantes un feedback sobre su aprendizaje y facilitar la reflexión sobre su progreso. Una de las principales razones para utilizar un instrumento en desarrollo es el impacto educativo que cualquier proceso de evaluación ejerce sobre quien aprende. La evaluación dirige el aprendizaje [28]. Así, con la utilización de este tipo de instrumentos, se enfatizan aspectos competenciales que de otra forma podrían pasar desapercibidos. En expresión radical, se dice que lo que no se evalúa, no existe o se devalúa.

\section{Puntos clave}

- El proceso de Bolonia centra el sistema educativo superior en 'resultados de aprendizaje'. Los resultados de aprendizaje se expresan en competencias evaluadas.

- El debate dominante de la educación centrada en los procesos deja de ser un fin en sí para convertirse en instrumental al de la adquisición de competencias.

- Las competencias como objetivo educativo ponen la adquisición de las actitudes, valores 
y comportamientos al mismo nivel que la de los conocimientos y habilidades, lo que debe conllevar cambios conceptuales, metodológicos y evaluativos. Educación deviene más que aprendizaje en 'aculturalización' o socialización secundaria. Es más que aprender, es cambiar.

- Los docentes deberán seguir siendo expertos en 'conocimiento', pero deberán desarrollar sus capacidades para ampliar esta función actual e incorporar otras nuevas, como mentores de apoyo al proceso de aprendizaje y modelos de comportamiento profesional.

- Las estructuras organizativas y presupuestarias de las facultades deberán adaptarse para la mejor consecución de sus fines: la adquisición, por parte de sus alumnos, de las competencias profesionales exigidas.

- La evaluación de la competencia profesional siempre debe combinar distintos instrumentos.

\section{Bibliografía}

1. The Association of Faculties of Medicine of Canada. The future of medical education in Canada (FMEC): a collective vision for MD education. URL: http://www.afmc.ca/ fmec. [29.06.2010].

2. Bologna Declaration. The European Higher Education Area. Joint Declaration of the European Ministers of Education (Bologna), 1999. URL: http://www.ond.vlaanderen.be/hogeronderwijs/bologna/about. [26.08.2010].

3. Smith SS, Dollase R. AMEE Guide No. 14. Outcomebased education: Part 2 -planning, implementing and evaluating a competency-based curriculum. Med Teach 1999; 21: 15-22.

4. Real Academia Española. Diccionario de la lengua española. 21. ${ }^{a}$ ed. Madrid: RAE; 1992.

5. Ley 16/2003 de Cohesión y Calidad del Sistema Nacional de Salud. BOE n. ${ }^{\circ} 128$, de 20 de marzo de 2003; p. 20567-88.

6. Ley $44 / 2003$ de Ordenación de las Profesiones Sanitarias. BOE n. ${ }^{\circ} 280$, de 21 de noviembre de 2003; p. 41442-58.

7. Consell Català d'Especialitats en Ciències de la Salut. Competències de professions sanitàries. Barcelona: Institut d'Estudis de la Salut; 2002.

8. General Medical Council. Good medical practice: regulating doctors. General Medical Council 2006. URL: http://www.gmc-uk.org/static/documents/content/ GMC_GMP_0911.pdf. [07.07.2010].

9. General Medical Council. Tomorrows Doctors: out- comes and standards for undergraduated medical education. General Medical Council 2009. URL: http://www. gmc-uk.org/static/documents/content/TomorrowsDoctors_2009.pdf. [07.07.2010].

10. Scottish Doctor. URL: http://www.scottishdoctor.org/ resources/scottishdoctor3.doc. [01.07.2009].

11. Palés J. La educación médica basada en las competencias finales del estudiante. Educ Med 2001; 4: 1.

12. Facultat de Medicina, Universitat de Barcelona. Competències que han d'adquirir els estudiants de Medicina durant els estudis de pregrau a la Facultat de Medicina de la Universitat de Barcelona. Barcelona: Universitat de Barcelona; 2003.

13. Palés J, Cardellach F, Estrach M, Gomar C, Gual A, Pons F, et al. Defining the learning outcomes of graduates from the medical school at the University of Barcelona (Catalonia, Spain). Med Teach 2004; 26: 239-43.

14. Palés J, Gual A, Gomar C, Estrach T. Acquisition of learning outcomes by students from the medical school of the University of Barcelona (Catalonia, Spain): a student Survey. Med Teach 2008; 30: 693-8.

15. Universitat de Lleida, Facultat de Medicina. Competències professionals a assolir durant el periode de formació de pregrau. Lleida: Universitat de Lleida; 2003.

16. Cátedra Educación Médica. Universidad Complutense de Madrid-Fundación Lilly. Competencias para el grado de Medicina de la Universidad Complutense de Madrid; 2008.

17. Prat J, Carreras J, Branda L, Miralles R, Fenoll RM, Rodríguez $\mathrm{S}$, et al. Competències professionals bàsiques comunes dels llicenciats en Medicina formats a les universitats de Catalunya. Barcelona: Agència per a la Qualitat del Sistema Universitari de Catalunya; 2004.

18. Wojtczak A, Schwarz MR. Minimum Essential requirements and standards in medical education. Med Teach 2000; 22: 555-63.

19. MEDINE. The Tuning learning outcomes/competences for primary medical degrees in Europe (2007). URL: http://www.tuning-medicine.com. [01.07.2010].

20. MEDINE. WFME global standards for quality improvement in medical education: European specifications. Copenhagen: WFME; 2007.

21. Conferencia Nacional de Decanos de Facultades de Medicina Españolas. Libro Blanco. Titulación de Medicina. URL: http://www.um.es/medicina/lic-medicina/libroblanco.pdf. [27.08.2010].

22. Orden ECI/332/2008, de 13 de febrero, por la que se establecen los requisitos para la verificación de los estudios universitarios oficiales que habiliten para el ejercicio de la profesión de médico. BOE n. ${ }^{\circ} 40$, de 15 de febrero de 2008; p. 8351-5.

23. Project ACGME. URL: http://www.acgme.org/outcome/ comp/compCPRL.asp. [27.08.2010]. 
24. CanMeds. URL: http://www.deptmedicine.utoronto.ca/ CanMeds. [27.08.2010].

25. Nolla-Domenjó M. La evaluación en educación médica. Principios básicos. Educ Med 2009; 12: 223-9.

26. Ginsburg S, McIlroy J, Oulanova O, Eva K. Toward authentic clinical evaluation: pitfalls in the pursuit of competency. Acad Med 2010; 85: 780-6.
27. Leach DC. Six competencies and the importance of dialogue with the community. ACGME e-bulletin 2006. URL: http://www.acgme.org/acWebsite/bulletin-e/ebu_ index.asp. [06.07.2010].

28. Wood D. Formative assessment. Edinburgh: Association for the Study of Medical Education; 2007. 